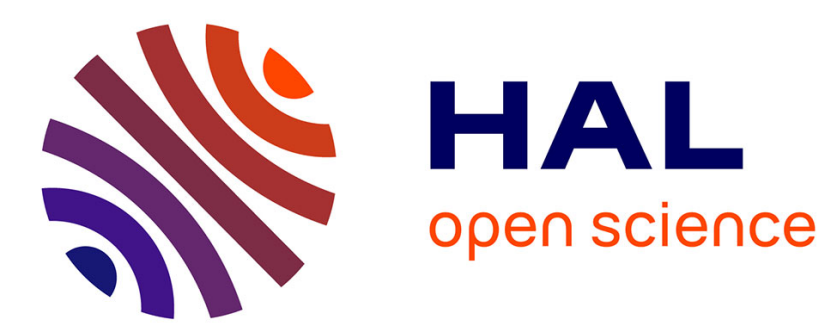

\title{
Magnetic quantum structures
}

F. Himpsel

\section{- To cite this version:}

F. Himpsel. Magnetic quantum structures. Journal de Physique IV Proceedings, 1994, 04 (C9), pp.C9-75-C9-82. 10.1051/jp4:1994909 . jpa-00253469

\section{HAL Id: jpa-00253469 https://hal.science/jpa-00253469}

Submitted on 1 Jan 1994

HAL is a multi-disciplinary open access archive for the deposit and dissemination of scientific research documents, whether they are published or not. The documents may come from teaching and research institutions in France or abroad, or from public or private research centers.
L'archive ouverte pluridisciplinaire HAL, est destinée au dépôt et à la diffusion de documents scientifiques de niveau recherche, publiés ou non, émanant des établissements d'enseignement et de recherche français ou étrangers, des laboratoires publics ou privés. 


\title{
Magnetic quantum structures
}

\author{
F.J. Himpsel
}

IBM Research Division, Thomas J. Watson Research Center, P.O. Box 218, Yorktown Heights, NY 10598, U.S.A.

\begin{abstract}
A class of materials is discussed, where quantum effects have macroscopic consequences. These are magnetic multilayers with spacers down to a few atomic layers. It is shown how quantization perpendicular to the layers is connected with oscillatory magnetic coupling, which in turn is important for obtaining "giant" magnetoresistance. This effect is being applied towards the fabrication of magnetoresistive reading heads for magnetically-stored data. The capabilities of various synchrotron radiation techniques for studying such nanometer scale systems are discussed, e.g. angle-resolved, spinpolarized photoemission, magnetic circular dichroism and core level fluorescence. Possible extensions towards lateral superlattices and quantum wires are pointed out, where a stepped surface is acting as template.
\end{abstract}

\section{INTRODUCTION}

Magnetic multilayers have generated a lot of interest recently by their unusual properties, such as oscillatory magnetic coupling and "giant" magnetoresistance (Ref.s 1-8). The former is a textbook example of the RKKY interaction in a lattice, the latter has applications in magnetic recording. The state-of-the-art in magnetic reading heads uses the magnetoresistance in permalloy, which is only a $2 \%$ effect. Multilayer structures offer an order of magnitude larger changes in resistance, thus enhancing the sensitivity to the signal from a magnetically-stored bit pattern. It would be very useful to be able to predict what type of coupling (parallel or antiparallel) a magnetic multilayer will exhibit, and how many spacer layers it takes to achieve a certain coupling. Spacer layers can be as thin as $10 \AA$, i.e. adding only a few atomic layers can affect the macroscopic magnetoresistance of the whole structure. Going farther out towards the limits of magnetic recording density one has to worry about the magnetic properties of small particles in the storage media. Although we are still about four orders of magnitude away from the superparamagnetic limit, where particles flip their magnetization spontaneously at room temperature, there are practical hurdles to be overcome before reaching the theoretical limit. They are intimately tied to the electronic structure of segregated ternary alloys (e.g. CoPtCr) which incorporate magnetic particles of a few hundred $\AA$ in size. 


\section{GIANT MAGNETORESISTANCE IN MULTILAYERS}

Before getting into the electronic structure of magnetic multilayers it is useful to understand the intended electronic properties, such as giant magnetoresistance (Ref.s 6-8). Fig. 1 (Ref. 9) gives a highly-simplified visualization of bulk and interface phenomena that affect the electrical resistance in a magnetic field. The popular model structure shown in Fig. 1 contains two magnetic layers separated by a nonmagnetic spacer. 'The giant magnetoresistance effect occurs only if the two magnetic layers prefer the unusual configuration with opposite magnetization directions. Then an external field is able to change the magnetic configuration by forcing both magnetizations to be parallel. The idea is to specifically design such materials, either by choosing the proper spacer layer thickness or by adding a fourth, magnetic layer to fix the magnetic orientation of one layer and let the other layer be switched by the external field (so-called spin valve design, Ref. 5). Understanding the magnetic coupling across the spacer layer is essential for this purpose. The observed lowering of the electrical resistivity with the external field can be rationalized by a simple model where one magnetic layer acts as a polarizer by generating a polarized current of majority spin electrons at the Fermi level, the other as analyzer by transmitting preferably majority spin electrons. The fact that majority spin electrons are the preferred charge carriers in magnetic materials can be explained by their longer mean free path compared to minority spin electrons at the Fermi level. The majority spin d-band is filled in many ferromagnets (e.g. Co and $\mathrm{Ni}$ ), making the band structure similar to that of a noble metal, where only a low density of $s, p$ states is available at the Fermi level to scatter electrons by electron-hole pair production. The minority spin band structure, on the other hand, has a much higher density of d-states at the Fermi level, producing more electron-hole pairs. (Note that spin-flip scattering is much less probable, such that the majority and minority spin band structures can be considered separately). In addition to this bulk effect, the interfaces themselves exhibit strongly spin-dependent reflectivities, giving rise to a large fraction of the observed magnetoresistance. Although structures with the current perpendicular to the layers, as in Fig. 1, exhibit very large magnetoresistance (Ref.s 10,11), the structures used in practice have the current parallel to the layers, in order to obtain reasonable resistivities. That generates extra complications though (Ref. 6) since the non-magnetic spacer tends to short out the magnetic layers. There is ample room for innovative ideas of new structures that optimize the desired magnetoresistive effect. For example, segregated magnetic particles in noble metals exhibit similar magnetoresistance effects.

Giant Magnetoresistance: Interface Effect

Field on:

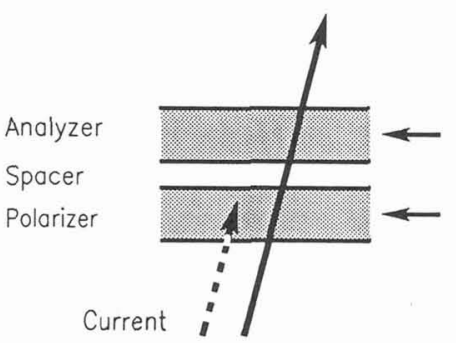

Field off:

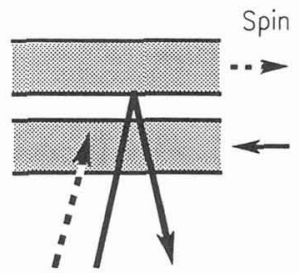

Giant Magnetoresistance: Bulk Effect

Field on:

Field off:

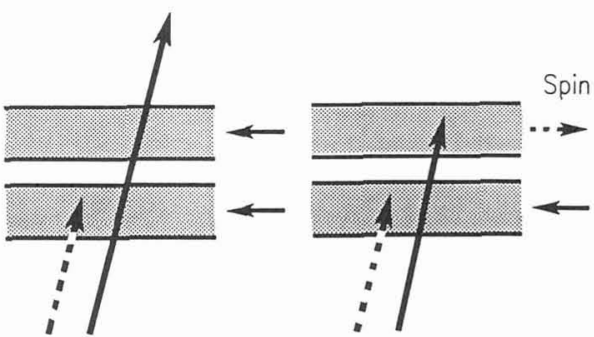

Fig. 1 Two possible effects contributing to the giant magnetoresistance in a spin valve. The bulk effect is based on the shorter mean free path for minority spins, the interface effect is caused by preferential scattering of minority electrons at the interface. Typical dimensions are $100 \AA$ for the thickness of the ferromagnetic layers (hatched) and $20 \AA$ for the nonmagnetic spacer (Ref. 9). 


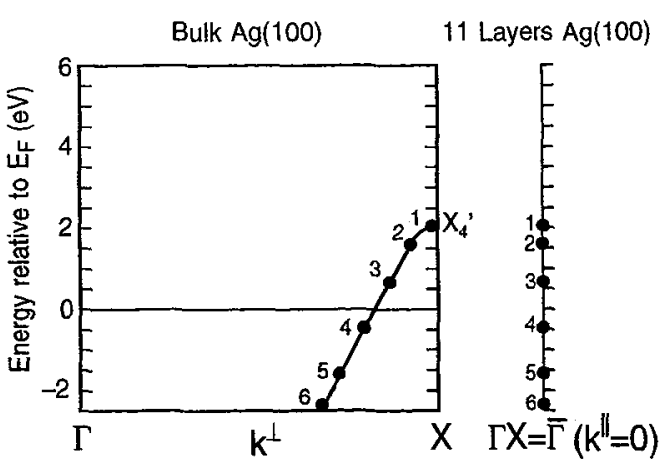

Fig. 2 Discretization of bulk bands in a thin film along the direction perpendicular to the surface $\mathrm{k}^{\perp}$, obtained from local density calculations. The number of discrete states per band corresponds to the number of atomic layers in the film (Ref. 12).

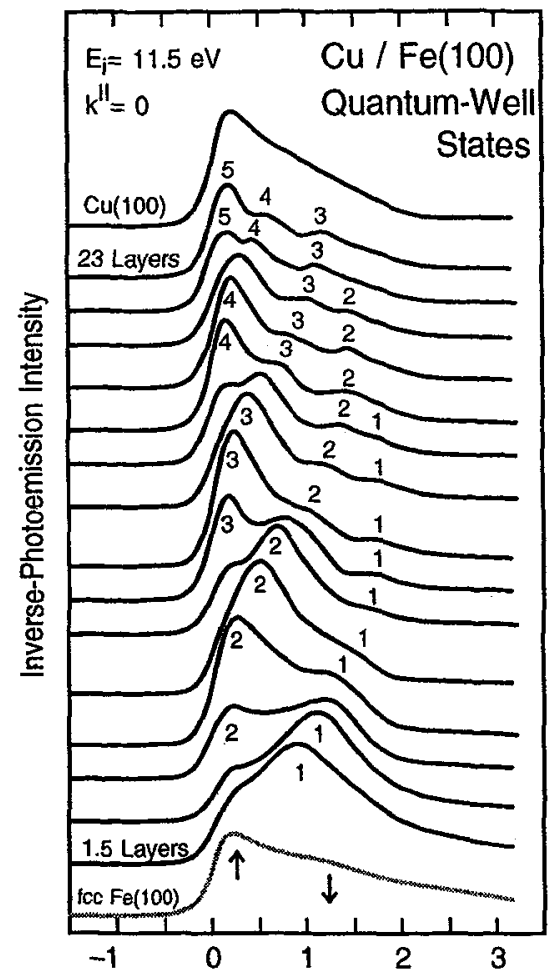

Energy relative to $E_{F}(e V)$

Fig. 3 Thickness dependence of inverse photoemission spectra for $\mathrm{Cu}$ films on $\mathrm{fcc}$ $\mathrm{Fe}(100)$ at $\mathrm{k}^{\|}=0$. The $\mathrm{s}, \mathrm{p}$-band continuum (top) is split up into the numbered quantum well states due to discretization of $\mathrm{k}^{\perp}$ in a thin film (see Fig. 2). Cu on fcc $\mathrm{Co}(100)$ behaves similarly (Ref. 13).

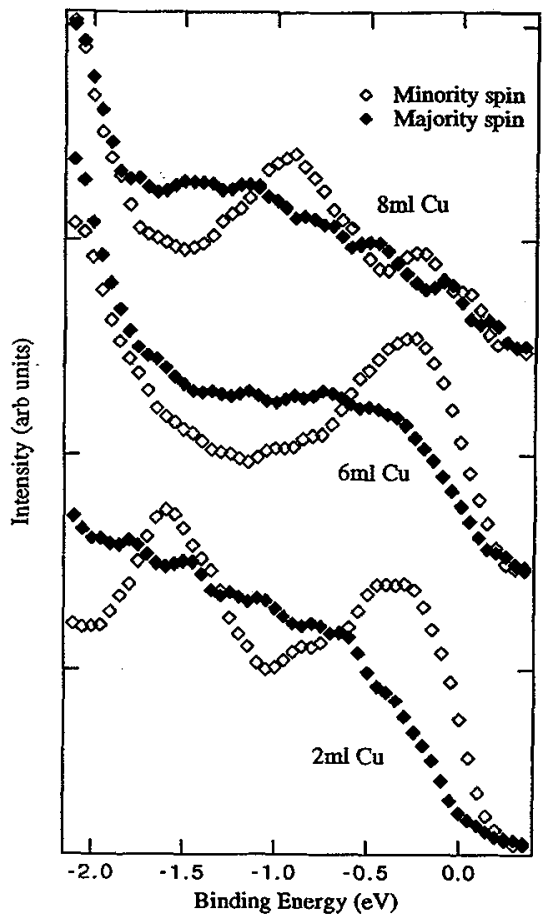

Fig. 4 Spin-polarized photoemission spectra of occupied quantum well states for $\mathrm{Cu}$ on fcc $\mathrm{Co}(100)$, showing that these states are spin-polarized, and thus can mediate magnetic coupling (Ref. 14; see also Fig. 5).

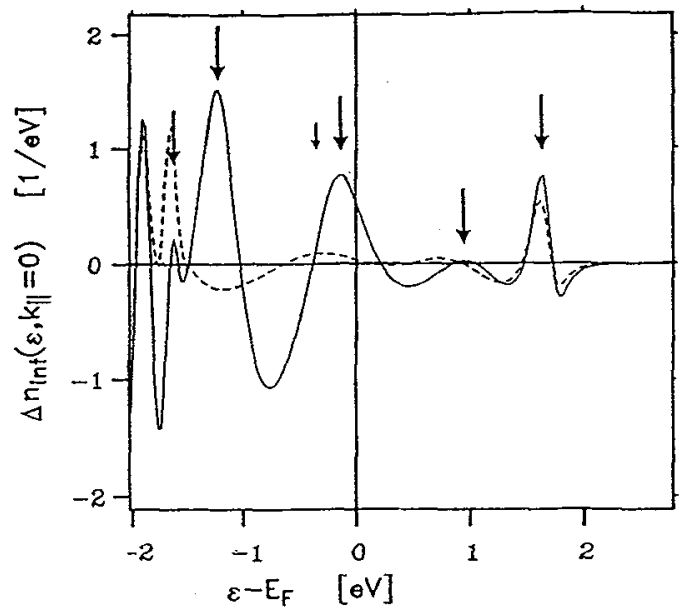

Fig. 5 First principles calculation of the spin-dependent density of states for a 6 layer $\mathrm{Cu}$ film on a $\mathrm{Co}(100)$ layer. Several quantum well states are seen (arrows) with predominantly minority spin polarization (full line) (Ref. 16). 


\section{MAGNETIC QUANTUM WELL STATES}

In the search for the electronic states that cause thickness-dependent oscillations in the magnetic coupling of multilayers one has to understand electronic states of thin films. As Fig. 2 (Ref. 12) shows, the continuum bulk band dispersion becomes discretized along the direction perpendicular to the interface when electrons are confined to a thin film. The number of discrete states per band corresponds to the number of atomic layers in the film. This discretization can be seen for thin $\mathrm{Cu}(100)$ films in Fig. 3 (Ref. 13), which have a band structure very similar to the $\mathrm{Ag}(100)$ film calculated in Fig. 2. At first one would expect that no magnetic information is transferred across the non-magnetic spacer layer by quantizing its energy bands. However, these discrete quantum well states are spin-polarized (Refs. 14,15), as evidenced in the spinpolarized photoemission spectra in Fig. 4 (Ref. 14). Since spin detection involves a loss of 3-4 orders of magnitude intensity and since tunability is required to enhance the transition from the s,p-band, this type of experiment is a prime application of undulator-based synchrotron light sources. The observed minority spin polarization is also seen in state-of-the-art band calculations (Fig. 5, Ref. 16). Spin-polarization of quantum well states in a nonmagnetic spacer layer can be rationalized rather easily by using a simple interferometer model (Fig. 6, Refs. 12,13). The $\mathrm{Cu}$ spacer layer is considered as a Fabry-Perot interferometer with the $\mathrm{Cu} / \mathrm{Co}$ and the $\mathrm{Cu} /$ vacuum interfaces as electron mirrors. A quantum well state corresponds to a standing wave, i.e. an interferometer fringe. When changing the thickness of the Cu spacer layer in Fig. 6, such interferometer fringes appear with a period of half an clectron wavelength. The $\mathrm{Cu} / \mathrm{Co}$ interface has a spin-dependent reflectivity and phase shift due to the spin-dependent band structure of Co. Therefore, the appearance of fringes becomes spin-dependent.

For states relevant to magnetic coupling and magnetotransport one has to focus on the region within a few kT around the Fermi level, as done in Fig. 6. Indeed the density of states at the Fermi level exhibits periodic maxima due to the appearance of quantum well states (top, Ref. 13). The spin-polarization also oscillates with the same period (middle, Ref. 14). This periodicity is identical to the magnetic coupling period as measured by the Kerr effect (bottom, $\mathrm{Ref}$. 4). The period is about 6 atomic layers for $\mathrm{Cu} / \mathrm{Co}(100)$, and can go down to 2 atomic layers in other systems, making the switch between ferromagnetic and antiferromagnetic coupling truly a matter of a single atomic layer.

In order to visualize the process of magnetic coupling it would be interesting to have a picture of the spin density distribution in the $\mathrm{Cu}$ spacer layer. Calculations readily provide this quantity (Fig. 7, Ref. 17), and there is some experimental access to it by using circular dichroism from core levels. By selecting the $\mathrm{Co} 2 \mathrm{p}$ and $\mathrm{Cu} 2 \mathrm{p}$ core level and using sum rules involving the angular and spin magnetic moment (Ref. 18) the average moments in the Co and Cu layers can be determined. Varying the $\mathrm{Cu}$ layer thickness one obtains some depth information, as shown in Fig. 7. Such circular dichroism experiments on a weakly-polarized noble metal have to detect small absorption changes of a few $\%$. They can only be done with intense, tunable and polarized synchrotron light sources.

\section{LATERAL SUPERLATTICES}

Multilayers represent just a specialized type of quantum structure. Looking at more general structures one arrives at lateral superlattices, quantum wires, and quantum dots. At this point in time one can only speculate whether or not such structures will provide interesting and useful phenomena. For example, one might consider a new magnetoresistive structure consisting of a lateral superlattice of ferromagnetic stripes separated by noble metal stripes. In this structure the current can flow perpendicular to the interfaces, where an enhancement of a factor of three 

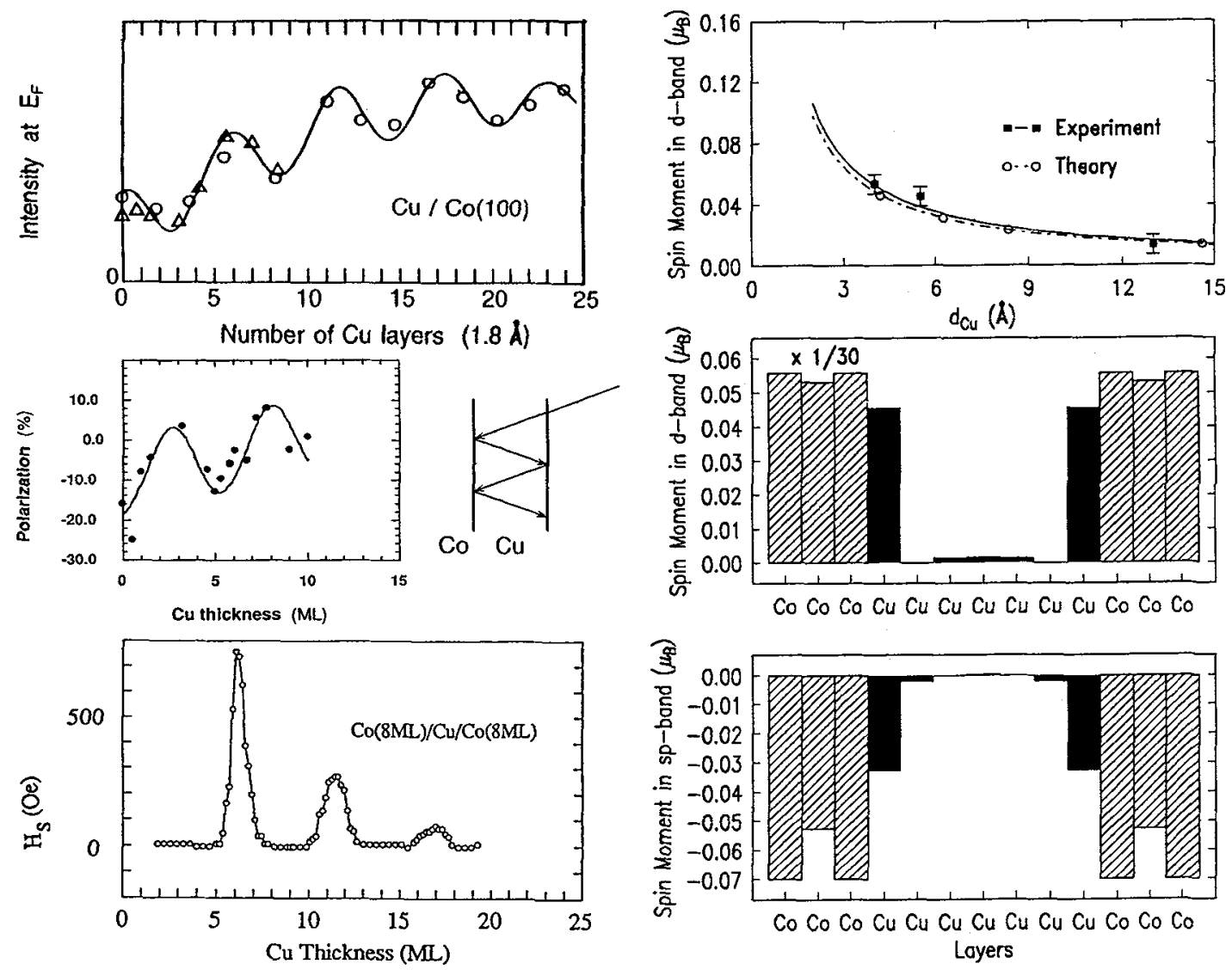

Fig. 6 Connection between oscillations in the density of states the Fermi level (top, Ref. 13), the spin-polarization (middle, Ref. 15) and oscillatory magnetic coupling (bottom, Ref. 4). The maxima in the switching field $\mathrm{H}_{\mathrm{s}}$ for the Kerr effect correspond to antiparallel coupling, the zeroes to ferromagnetic coupling. A simple interferometer model is also shown (Refs. 12, 13), explaining the oscillations as electron interface fringes.

Fig. 7 Determination of average spin magnetic moments in the $\mathrm{Cu}$ spacer layers of sputtered $\mathrm{Co} / \mathrm{Cu}$ multilayers, using magnetic circular dichroism at the $\mathrm{Cu} 2 \mathrm{p}$ core absorption edge. The data for varjous $\mathrm{Cu}$ spacer thicknesses are compared to a first principles calculation, which provides the spin moments at individual atomic layers (Ref. 17). The Co d-band moment is scaled down by a factor of 30 compared to $\mathrm{Cu}$.

to ten has been found for the magnetoresistance, but remain parallel to the surface in order to keep the resistance at workable levels.

How can such lateral superlattices be fabricated with atomic precision? Borrowing from semiconductor technology (Ref. 19) one can try to use the regular array of steps at a vicinal surface as template. The steps may be decorated by evaporating a metal that secks out high binding energy sites at a step, and if the surface energetics are right, additional rows of adatoms will also incorporate at the step edges and produce a step flow growth mode. Stopping growth at submonolayer coverage and switching to a different metal will then provide the desired striped structure. First experiments on metal substrates are under way to test the feasibility of such an approach (Fig. 8, Ref. 20). As one can sec, the $\mathrm{Cu}$ "stripes" produced in this fashion on stepped 


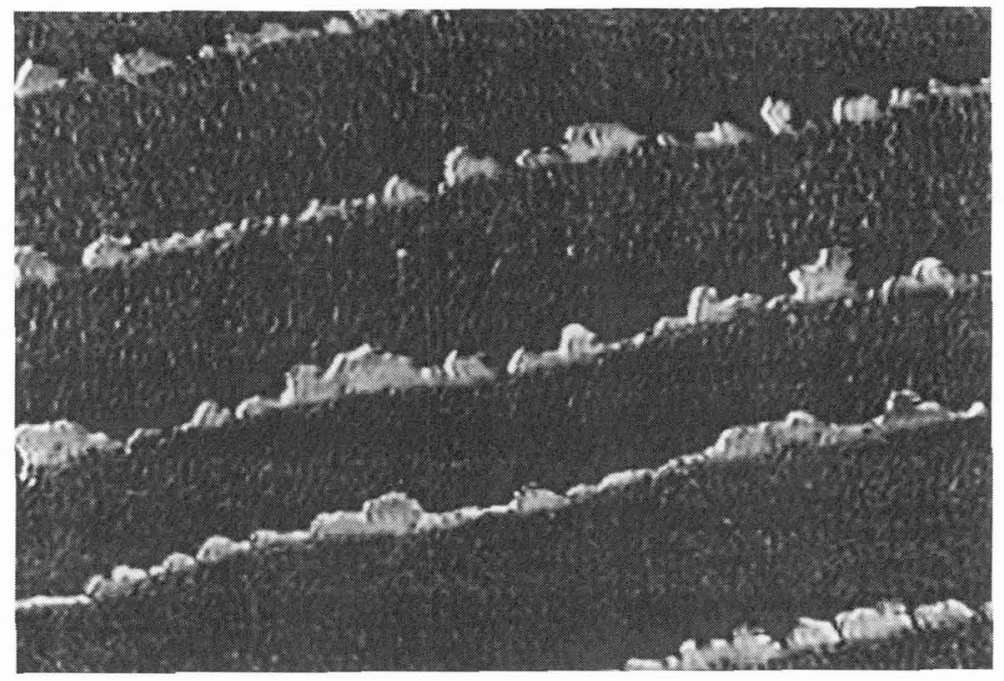

Fig. 8 STM picture of "quantum wires" of $\mathrm{Cu}$ (bright) attached to steps at a W(110) surface (Ref. 20 ). The field of view is $100 \mathrm{~nm} \times 70 \mathrm{~nm}$.
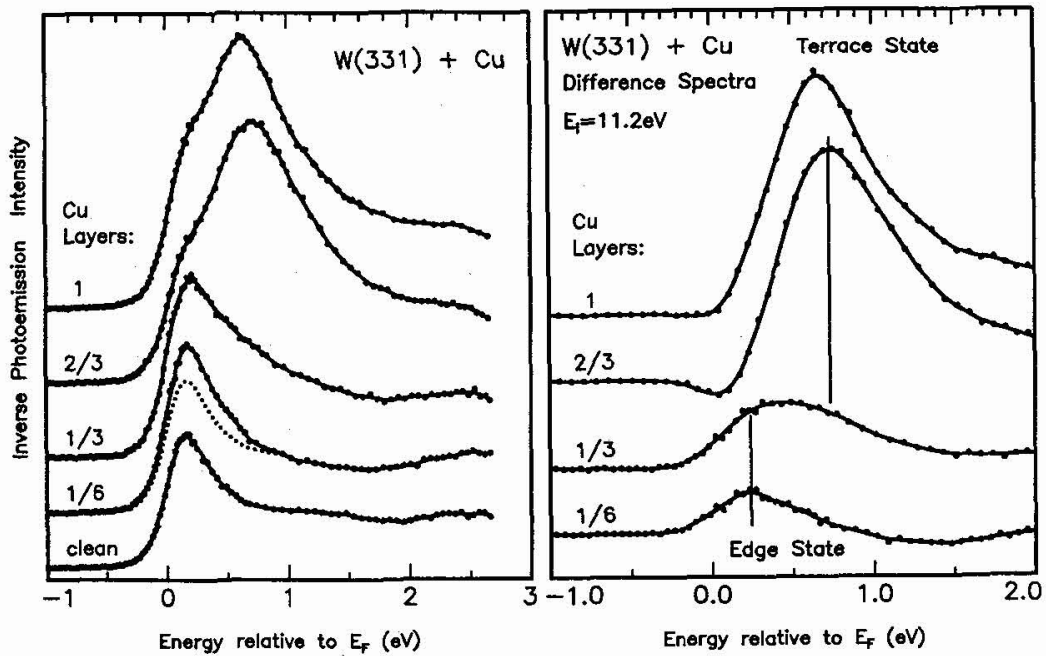

Fig. 9 Inverse photoemission spectra of $\mathrm{Cu}$ on W(331), a highly-stepped W(110) surface. $\mathrm{Cu}$ atoms attached to a step edge exhibit a level shift of $0.5 \mathrm{eV}$ for a $\mathrm{Cu}$-induced state at $0.7 \mathrm{eV}$. Raw data are on the left, the $\mathrm{Cu}$-induced signal on the right. A coverage of $1 / 6$ layer corresponds to a single row of $\mathrm{Cu}$ atoms per step (Ref. 21). The instrumental resolution is $0.25 \mathrm{eV}$.

$\mathrm{W}(110)$ are still rather ragged, but their width of $\approx 1 \mathrm{~nm}$ is well below the dimensions reachable with lithography or with STM-induced chemical vapor deposition. At the atomic level, i.e. for the first string of $\mathrm{Cu}$ atoms attached to a $\mathrm{W}$ step edge, we have good reason to believe that the $\mathrm{Cu}$ atoms indeed form a monoatomic string, since that is the most efficient way to lower the high step energy of $\mathrm{W}$. Indeed, when looking at the electronic states of this system one finds a $\mathrm{Cu}$ - 
induced state that exhibits an energy shift of $\approx 0.5 \mathrm{eV}$ when the $\mathrm{Cu}$ coverage is reduced to the single row limit (Fig. 9, Ref. 21; note that the W(331) surface in Fig. 9 has a much higher step density than the nearly flat W(110) surface in Fig. 8) No such shift is seen on a flat W substrate.

To explore the electronic and magnetic structure of such "quantum wires" and "quantum stripes" one needs an atom-specific, background-free probe that suppresses the substrate signal. Core level fluorescence has these properties, but it also suffers from a cross section three orders of magnitude smaller than the competing Auger and photoemission processes. This holds for the sharpest core levels of the elements which all lie below $1 \mathrm{keV}$ binding energy. Thus we are driven again to an intense, tunable light source. It has just become feasible to take a core level fluorescence spectrum of a monolayer (Ref. 22) at an undulator beam line at the Advanced Light Source (ALS). Using spectrally-integrated core fluorescence to measure absorption or dichroism, one should be able to reach well below monolayer sensitivity. In addition, the signal from buried layers in realistic device structure can be obtained, due to the long penetration depth of the fluorescence photons. For example, the B monolayer in the ALS experiment (Ref. 22) was buried under $50 \AA$ of carbon. Such experiments promise to become an interesting part in the arsenal of techniques available at the new, third generation synchrotron light sources.

\section{REFERENCES}

[1] Baibich, M. N., Broto, J. M., Fert, A., Ngyuen van Dau, F., Petroff, F., Etienne, P., Creuzet, G., Friederich, A., and Chazelas, J., Phys. Rev. Lett. 61 (1988) 2472.

[2] Binasch, G., Grünberg, P., Saurenbach, F., and Zinn, W., Phys. Rev. B 39 (1989) 4828.

[3] Parkin, S. S. P., More, N., and Roche, K. P., Phys. Rev. Lett. 64 (1990) 2304; Parkin, S. S. P., Bhadra, R., and Roche, K. P., Phys. Rev. Lett. 66 (1991) 2152.

[4] Xiu, Z. Q., Pearson, J., and Bader, S. D., Phys. Rev. B 46 (1992) 8659.

[5] Dieny, B., Speriosu, V. S., Parkin, S. S. P., Gurney, B. A., Wilhoit, D. R., Mauri, D., Phys. Rev. B 43 (1991) 1297; Dieny, B., Humbert, P., Speriosu, V. S., Metin, S., Gurney, B. A., Baumgart, P., Lefakis, H., Phys. Rev. B 45 (1992) 806.

[6] Hood, R. Q. and Falicov, L. M., Phys, Rev. B 46 (1992) 8287.

[7] Camblong, H. E., Zhang, S., and Levy, P. M., Phys. Rev. B 47 (1993) 4735.

[8] Valet, T. and Fert, A., Phys. Rev. B 48 (1993) 7099.

[9] Ortega, J. E., Himpse], F. J., Mankey, G. J., and Willis, R. F., Mat. Res. Soc. Symp. Proc. 313 (1993) 143.

[10] Pratt, W. P., Lee., S.-F., Slaughter, J. M., Loloee, R., Schroeder, P. A., and Bass, J, Phys. Rev. Lett. 66 (1991) 3060.

[11] Gijs, M. A. M., Lenczowski, S. K. J., and Giesbers, J. B., Phys. Rev. Lett. 70 (1993) 3343.

[12] Ortega, J. E., Himpsel, F. J., Mankey, G. J., and Willis, R. F., J. Appl. Phys. 73 (1993) 5771.

[13] Ortega, J. E. and Himpsel, F. J., Phys. Rev. Lett. 69 (1992) 844; Ortega, J. E., Himpsel, F. J., Mankey, G. J., and Willis, R. F., Phys. Rev. B 47 (1993) 1540.

[14] Garrison, K., Chang, Y., and Johnson, P. D., Phys. Rev. Lett. 71 (1993) 2801.

[15] Carbone, C., Vescovo, E., Rader, O., Gudat, W., and Eberhardt, W., Phys. Rev. Lett. 71 (1993) 2805.

[16] Nordström, L., Lang, P., Zeller, R., and Dederichs, P. H., submitted to Phys. Rev. Lett.; I am grateful to the authors for a preprint of this work.

[17] Samant, M. G., Stöhr, J., Parkin, S. S. P., Held, G. A., Hermsmeier, B. D., and Herman, F., Phys. Rev. Lett. 72 (1994) 1112. Circular dichroism experiments have also been performed for the $1 \mathrm{~s}$ level of $\mathrm{Cu}$ and $\mathrm{Co}$. They probe the magnetic moment in the p-band; see: Pizzini, S., Giorgetti, C., Fontaine, A., Dartyge, E., Krill, G., Bobo, J. F., and Piecuch, M., Mat. Res. Soc. Symp. Proc. 313 (1993) 625. 
[18] Thole, B. T., Carra, P., Sette, F., and van der Laan, G., Phys. Rev. Lett. 68 (1992) 1943; Wu, R., Wang, D., and Freeman, A. J., Phys. Rev. Lett. 71 (1993) 3581; Carra, P., Thole, B. T., Altarelli, M., and Wang, X., Phys. Rev. Lett. 70 (1993) 694.

[19] Petroff, P. M., Gossard, A. C., and Wiegmann, W., Appl. Phys. Lett. 45 (1984) 620; Nötzel, R., Ledentsov, N. N., Däweritz, L., Hohenstein, M., and Ploog, K., Phys. Rev. Lett. 67 (1991) 3812 .

[20] Mo, Y. W., and Himpsel, F. J., Phys. Rev. B, submitted.

[21] Himpsel, F. J., and Ortega, J. E., Phys. Rev. B, submitted.

[22] Yurkas, J., Ellis, A. W., and Himpsel, F. J., Samant, M. G., Stöhr, J., Jia, J., Callcott, T. A., Ederer, D., Carlisle, J. A., Terminello, L. J., Perera, R. C. C., Bull. Am. Phys. Soc. 39 (1994) 371. 